\title{
TNF $\alpha$ modifica la respuesta de la frecuencia de batido ciliar a la carga viscosa, asociado a alteraciones en el calcio intracelular en células ciliadas respiratorias humanas
}

\author{
TNF $\alpha$ modifies ciliary beat frequency in response to viscous overload, \\ associated to changes in intracellular calcium in human respiratory ciliated cells
}

Claudia González G1', Trinidad Sánchez D², Alejandra Pérez S², Ximena Fonseca A', Manuel Villalón B².

\section{RESUMEN}

Introducción: Secreciones sinonasales patológicas y elevados niveles de factor de necrosis tumoral alfa (TNF $\alpha$ ) se han encontrado en mucosa sinusal de pacientes con sinusitis crónica. Las células ciliadas respiratorias tienen una reserva funcional que les permite autorregular su frecuencia de batido ciliar (FBC) en respuesta a cambios en la viscosidad, modificando los niveles de calcio intracelular $\left[\mathrm{Ca}^{+2}\right]_{i c^{*}}$

Objetivo: Nuestro objetivo es determinar si TNFa afecta el mecanismo de autorregulación y la homeostasis del calcio intracelular frente a cambios en la viscosidad.

Material y método: Cultivos primarios de explantes de tejido adenoideo. Registro de FBC mediante microfotodensitometría. Cultivos tratados con $T N F \alpha(10 \mathrm{ng} / \mathrm{ml}) 0$ control durante 24 y 48 horas. Se incrementó la viscosidad agregando dextrano 500 al $10 \%$ y $20 \%$. Se midió $\left[\mathrm{Ca}^{+2}\right]_{i c}$ en células cargadas con Fura $2 A M$.

Resultados: El tratamiento con TNF $\alpha$ por 48 horas produjo una significativa disminución de la FBC a baja viscosidad, aumento significativo de $\left[\mathrm{Ca}^{+2}\right]_{i c}$ y caída mayor de FBC en cultivos tratados con tapsigargina (bloqueador bomba calcio-ATPasa retículo). No se encontró diferencia a alta viscosidad.

Conclusión: Después de 48 horas de exposición a TNFo se observa un efecto negativo en el mecanismo de adaptación de las células ciliadas a un medio con baja viscosidad, probablemente secundario a cambios en la homeostasis del $\left[\mathrm{Ca}^{+2}\right]_{i c^{*}}$

Palabras clave: Frecuencia de batido ciliar, factor de necrosis tumoral alfa, TNF $\alpha$, viscosidad, calcio intracelular.

\section{ABSTRACT}

Introduction: Pathologic sinonasal secretions and elevated levels of tumor necrosis factor alpha (TNF $\alpha$ ) have been noted in sinus mucosa of patients with chronic sinusitis. Respiratory

1 Médico. Departamento Otorrinolaringología, Hospital Clínico Pontificia Universidad Católica de Chile.

2 Doctor en Ciencias. Departamento de Fisiología, Facultad de Ciencias Biológicas, Pontificia Universidad Católica de Chile. 
ciliated cells have a functional reserve that allows them to autoregulate their ciliary beat in response to the changes in viscosity, modifying intracellular calcium levels $\left[\mathrm{Ca}^{+2}\right]_{i c^{*}}$

Aim: Our goal was to determinate if TNF $\alpha$ affect this autoregulation to viscosity and calcium homeostasis.

Material and Method: Primary cultures from adenoid tissue explants. Ciliary beat frequency (CBF) was recorded using microphotodensitometry. Cultures were treated with $T N F \alpha(10 \mathrm{ng} / \mathrm{ml})$ or control during 24 and 48 hours. Viscosity was increased by adding dextran $50010 \%$ and $20 \%$. $\left[\mathrm{Ca}^{+2}\right]_{i c}$ was determined in cells loaded with fura$2 A M$.

Results: 48 hours treatment with TNF $\alpha$ produced a significant decrease in CBF at low viscosity, significant increase in $\left[\mathrm{Ca}^{+2}\right]_{i c}$ and greater decrese in $\mathrm{CBF}$ in cultures treated with thapsigargin (endoplasmic calcium-ATPase pump blocker).

Conclusions: After 48 hours of exposure to TNF $\alpha$ a negative effect in the adaptation mechanism to a low viscous media is observed in ciliated cells, probably secondary to changes in homeostasis of $\left[\mathrm{Ca}^{+2}\right]_{i c^{\circ}}$.

Key words: Ciliary beat frequency, tumor necrosis factor alpha, TNF $\alpha$, viscosity, intracellular calcium.

\section{INTRODUCCIÓN}

El epitelio de toda la vía aérea está cubierto por un epitelio ciliado, cuyos cilios baten constantemente, manteniendo constante el transporte mucociliar. Este último es un importante mecanismo de defensa que permanentemente remueve bacterias y partículas fuera de la vía aérea ${ }^{1}$. La velocidad de transporte mucociliar es determinada principalmente por la frecuencia de batido ciliar. Incrementos modestos de la FBC pueden resultar en grandes aumentos en la velocidad de transporte ciliar $^{2}$. La FBC es regulada por señales de diversa naturaleza ${ }^{3}$.

Las infecciones bacterianas tienen un efecto negativo sobre el transporte mucociliar, ya sea por acción de citoquinas o por cambio en las características viscoelásticas del moco (hipersecreción, disminución en el contenido de agua, cambio en la expresión de mucinas), generando un aumento en la viscosidad de las secreciones ${ }^{4}$. Las células ciliadas son capaces de mantener una FBC relativamente constante sobre un gran rango de viscosidades del medio eterno. Este mecanismo de autorregulación ha sido observado en epitelio de oviducto de hámster, tráquea humana y de conejo ${ }^{5}$. Probablemente es responsable de evitar el colapso del transporte mucociliar frente a alta viscosidad. Esta respuesta podría generarse localmente dentro de la célula y está acoplada a incrementos en $\left[\mathrm{Ca}^{+2}\right]_{\mathrm{ic}}{ }^{6}$.
El factor de necrosis tumoral alfa (TNF $\alpha$ ) es una citoquina proinflamatoria producida por diferentes tipos celulares incluyendo macrófagos, monocitos, linfocitos, queratinocitos y fibroblastos en respuesta a infección, inflamación, daño u otras alteraciones. Se ha observado un aumento en los valores basales de TNF $\alpha$ en secreciones nasales de pacientes con infecciones del tracto respiratorio alto ${ }^{7}$, y luego de exposición a antígeno en pacientes con rinitis alérgica ${ }^{8}$. Los niveles de TNF $\alpha$ son significativamente más altos en secreción nasal de pacientes con sinusitis crónica que pacientes normales ${ }^{9} \mathrm{y}$ se mantienen elevados en pacientes con sinusitis crónica con pobre respuesta a tratamiento médico, sugiriendo que la persistencia de mediadores de la inflamación hace a la mucosa más susceptible de infecciones y episodios recurrentes ${ }^{10}$. Su efecto sobre la frecuencia de batido ciliar in vitro es controvertido. Algunos autores señalan que a bajas concentraciones $(0,1$ y $1 \mathrm{ng} / \mathrm{ml})$ estimula la $\mathrm{FBC}$, mientras que en altas concentraciones $(10 \mathrm{ng} / \mathrm{ml})$, disminuye la $\mathrm{FBC}^{11}$. Por el contrario, otros han observado un efecto estimulador de la FBC luego de la exposición a $10 \mathrm{ng} / \mathrm{mL}^{\text {de }} \mathrm{TNF} \alpha^{12}$.

Actualmente se desconoce cómo la interacción entre cambios en la viscosidad del medio externo y liberación de citoquinas puede afectar el funcionamiento de la célula ciliada. Planteamos como hipótesis que los niveles elevados de TNF $\alpha$, observados en enfermedades crónicas de la vía aérea superior, 
tienen un impacto negativo sobre el transporte mucociliar, alterando el funcionamiento normal de las células ciliadas. El objetivo de este estudio es evaluar cómo TNF $\alpha$ afecta el mecanismo de autorregulación a la carga viscosa y $\left[\mathrm{Ca}^{+2}\right]_{\mathrm{ic}}$.

\section{MATERIAL Y MÉTODO}

\section{Muestras de tejido}

Se obtuvo tejido adenoideo de pacientes pediátricos (entre 3 y 12 años de edad) sometidos a adenoidectomía por patología obstructiva (hipertrofia adenoidea 0 adenoamigdalina), previa autorización de los padres. El diseño del estudio y el consentimiento informado fueron revisados y aprobados por el Comité de Ética de la Pontificia Universidad Católica de Chile.

El tejido adenoideo fue colocado en solución de suero fisiológico para remover los coágulos y luego en solución salina balanceada Hank's (HBSS) a pH 7,4, suplementada con antibióticos (estreptomicina $10 \mu \mathrm{g} /$ $\mathrm{ml}$, penicilina $\mathrm{G} 100 \mathrm{U} / \mathrm{ml}$, amfotericina B $0,125 \mu \mathrm{g} / \mathrm{ml}$ ).

En el laboratorio, las muestras de tejido adenoideo fueron lavadas con DMEM-F12 suplementado con antibióticos para remover restos de sangre, luego fueron incubadas toda la noche a $4^{\circ} \mathrm{C}$ en medio DMEM F12 con antibióticos y pronasa $0,05 \% \mathrm{p} / \mathrm{v}$. Al día siguiente el tejido fue transferido a un contenedor con medio DMEM-F12 con suero fetal bovino (SFB) $5 \%$ y el epitelio se removió mecánicamente.

\section{Cultivos primarios}

Los cultivos primarios se realizaron con el método introducido por Verdugo y cols $^{13}$, que permite obtener monocapas confluentes de células epiteliales, descrito previamente ${ }^{14}$. Brevemente, el epitelio adenoideo es cortado en trozos de 2-4 mm, que son depositados en cubreobjetos pretratados con gelatina al $0,1 \%$, y éstos en cámaras de Rose. Las cámaras de Rose se llenaron con $2 \mathrm{ml}$ de medio NHS que contiene $10 \%$ de suero de caballo inactivado por calor (ph 7,2-7,4) y se mantienen en una incubadora a $37^{\circ} \mathrm{C}$.

El medio de las cámaras se renueva cada 48 horas. Después de aproximadamente 5 días se forma una monocapa de células ciliadas que presentan batido ciliar sincronizado. Cuando esto ocurre el cultivo se considera listo para estudio.

\section{Inmunofluorescencia TNF receptor 1 (TNFR1) y TNF receptor 2 (TNFR2)}

Los cultivos celulares fueron fijados y procesados por la técnica previamente descrita ${ }^{15}$. Las secciones fueron incubadas ya sea con anticuerpo policlonal TNFR1 goat-anti rat 1:50 (Santa Cruz Biotechnology, Santa Cruz, CA, USA) o anticuerpo policlonal TNFR2 goat anti-rat 1:100 (Santa Cruz Biotechnology) durante 2 horas a $37^{\circ} \mathrm{C}$. Posteriormente fueron incubadas durante 1 hora a $37^{\circ} \mathrm{C}$ con el anticuerpo secundario donkey anti-goat HRP (Santa Cruz Biotechnology). A continuación fueron incubadas con Avidin Biotin Enzyme Reagent (Vector Laboratories, Burlingame, CA, USA) durante 1 hora a temperatura ambiente y la reacción fue visualizada por la adición de 3,3'- tetrahidrocloruro diaminobencidina (DAB; $1,2 \mathrm{mg} / \mathrm{ml}$ ).

\section{Detección del batido ciliar con la técnica de microfotodensitometría}

La técnica descrita previamente ${ }^{14}$, consiste en el análisis de las fluctuaciones de luz que genera el movimiento de los cilios y que son detectadas por un fotodiodo asimétrico montado en el plano fotográfico del microscopio de fase ${ }^{16}$. El sistema que actualmente está montado en el laboratorio, permite la detección de la frecuencia en células ciliadas individuales que contienen aproximadamente 50100 cilios por célula.

\section{Tratamiento con TNF $\alpha$}

Una vez que los cultivos alcanzaron confluencia, éstos fueron incubados con factor de necrosis tumoral alfa recombinante humano (hrTNF $\alpha$ ) (Sigma-Aldrich) $(10 \mathrm{ng} / \mathrm{ml}) 0$ solución control (medio de cultivo) por 24 ó 48 horas. Posteriormente se midió FBC.

\section{Tratamiento con tapsigargina}

Se midió FBC basal por 10 minutos, luego se agregó tapsigargina (bloqueador de la bomba cal- 
cio-ATPasa del retículo endoplásmico) en concentración final de 2,5 $\mu \mathrm{M}$. Se realizaron mediciones de FBC y sólo se agregó la solución con dextrano una vez que la FBC volvió a la basal.

\section{Medición de frecuencia de batido ciliar}

Se realizaron lavados del cultivo con HBSS y se midió la frecuencia de batido ciliar en el microscopio a $35^{\circ} \mathrm{C}$. Se seleccionaron dos o tres células con una frecuencia basal promedio para ese cultivo, con buena señal y se realizaron mediciones de FBC cada un minuto durante cinco minutos. Cuando la frecuencia permanecía estable se realizaron los experimentos.

\section{Incremento en la viscosidad del medio externo}

Se agregó al cultivo dextrano (dextran 500 Sigma Aldrich) diluido en HBSS, en concentración final de $5 \%, 10 \%, 15 \%, 20 \%$ y $25 \%$ y se realizaron mediciones de FBC durante 20 minutos. Posterior a esto se lavó el medio con HBSS y se controló FBC por cinco minutos, para evaluar que la droga utilizada no haya alterado la función celular durante el experimento.

\section{Mediciones de niveles de calcio intracelular o citosólico $\left[\mathrm{Ca}^{2+}\right]_{i}$}

Se realizaron mediciones de $\left[\mathrm{Ca}^{2+}\right]_{\text {, }}$ utilizando la técnica de espectrofluorometría descrita previamente ${ }^{17}$. Los cultivos con actividad ciliar espontánea a $37^{\circ} \mathrm{C}$ fueron cargados con el indicador fluorescente de $\left[\mathrm{Ca}^{2+}\right]_{i}$ Fura-2AM (Invitrogen) (1.5 $\mu \mathrm{M}$ in HBSS) durante 45 minutos a $37^{\circ} \mathrm{C}$. Las células fueron excitadas a 349 y $380 \mathrm{~nm}$ en un microscopio Nikon Diaphot equipado con un espectrofluorómetro Photon Technology International (Lawrenceville, NJ) para análisis de razón entre ambas fluorescencias. Después de 10 minutos de estabilización, la intensidad de razón fue continuamente grabada y los cultivos fueron estimulados con solución de dextrano 10\%. La emisión de la intensidad de fluorescencia fue detectada con un fotomultiplicador y analizada utilizando un programa de análisis de fluorescencia (FELIX versión 1.1).

\section{Análisis estadístico}

Los datos son expresados como promedio $\pm \mathrm{EE}$ (error estándar). Los datos de FBC fueron analizados después de la transformación del arcoseno de los porcentajes. Las comparaciones estadísticas entre las diferentes condiciones experimentales fueron realizadas utilizando test $T$ y ANOVA con el programa GraphPad Prism4. El criterio para diferencia estadísticamente significativa fue $p<0,05$.

\section{RESULTADOS}

Expresión de los receptores TNF $\alpha R 1$ y TNF $R 2$ en cultivo: La tinción inmunohistoquímica indicó que el TNFaR1 está presente en células ciliadas en cultivo. La marca se concentró en el citoplasma de las células ciliadas. No se observó inmunorreactividad positiva para TNF $\alpha$ R2. Las secciones tratadas en ausencia de anticuerpo primario no mostraron inmunorreactividad (Figura 1).

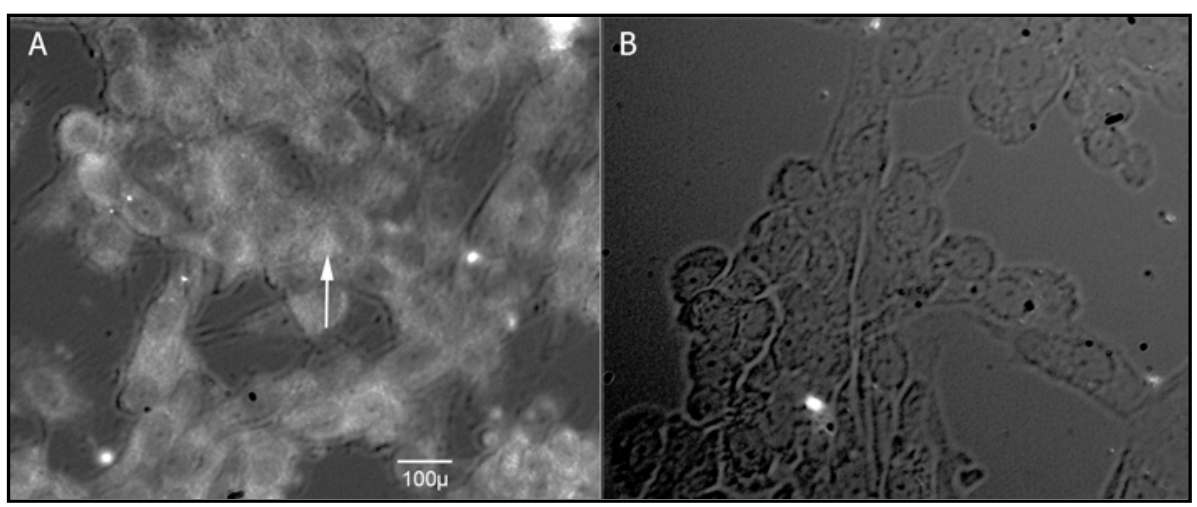

Figura 1. Las células ciliadas respiratorias humanas en cultivo expresan el receptor 1 de TNF $\alpha$ (TNFR1)

Microfotografía de una inmunofluorescencia indirecta de un cultivo primario de células ciliadas. A) TNFR1, se observa inmunorreactividad positiva en el citoplasma celular (flecha). B) Condición control en ausencia de anticuerpo primario. 
FBC y carga viscosa: La exposición de células ciliadas humanas a incremento en la carga viscosa del medio externo produjo una disminución en la FBC hasta alcanzar un nuevo valor estable dentro de los primeros 10 minutos (Figura 2a). La FBC disminuyó aproximadamente $20 \%$ dentro del rango de 4,75 a $37 \mathrm{cP}$ (soluciones con dextrano $5 \%$ a $15 \%$ ), y $30 \%$ en el rango de 73 a 122,4 cP (soluciones con dextrano 20\%-25\%). En este último intervalo, no se observó mayor caída en la FBC a pesar del incremento en la viscosidad (Figura 2b).

Efecto del tratamiento con TNF $\alpha$ (24 horas) sobre la respuesta a carga viscosa: El incremento en la carga viscosa con solución de dextrano $10 \%$ produjo una caída máxima en la FBC de $11,49 \% \pm 2,61$ en los cultivos tratados con TNF $\alpha$ y $16,81 \% \pm 6,78$ en los controles ( $p$ $>0,05$ ). La solución con dextrano $20 \%$ produjo una caída máxima en la FBC de $29,21 \% \pm 4,57$ en los cultivos tratados con TNF $\alpha$ y $29,13 \% \pm 3,85$ en los controles ( $p$ $>0,05$ ) (Figura 3). No se observó efecto significativo del
Efecto del tratamiento con TNF $\alpha$ (48 horas) sobre la respuesta a carga viscosa: En los cultivos tratados por 48 horas con TNF $\alpha$ frente al incremento en la viscosidad con dextrano $10 \%$, se observó una caída máxima en la FBC de $22,11 \% \pm 1,91$, a diferencia de los controles en que la caída fue de $12,2 \% \pm 4,21$. La diferencia entre el área bajo la curva de ambos grupos durante la exposición a dextrano fue estadísticamente significativa $p<0,05$.

La exposición a dextrano 20\% determinó una disminución máxima en la FBC de 25,95\% $\pm 6,24$ en los cultivos tratados y $22,45 \%$ en los controles. No se observó diferencia significativa en el área bajo la curva (Figura 4).

Efecto del tratamiento con TNF $\alpha$ (48 horas) sobre los niveles de $\left[\mathrm{Ca}^{2}\right]_{i}$ en respuesta a carga viscosa: El aumento de la viscosidad extracelular con dextrano $10 \%$ produjo un rápido y transitorio incremento en los niveles de $\left[\mathrm{Ca}^{2+}\right]_{\text {ic }}$ (Figura 5a), tanto en controles como

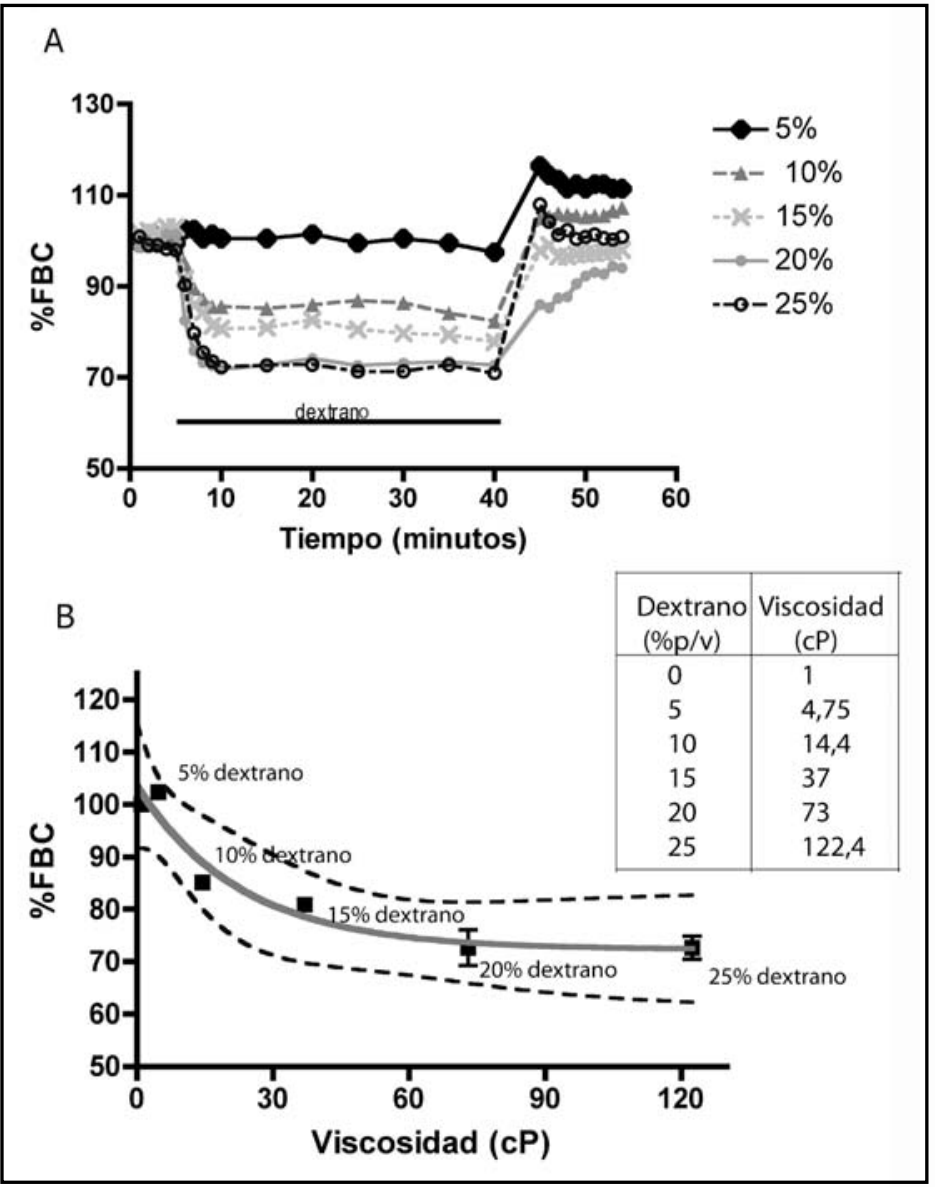

Figura 2. Las células ciliadas en cultivo presentan un mecanismo de adaptación a la carga viscosa.

A) Curso temporal del efecto de la carga viscosa sobre la FBC. Cultivos expuestos a diferentes concentraciones de dextrano. Los datos corresponden a valores promedio de FBC y se expresan como porcentaje respecto a la FBC basal (100\%). B) Relación entre la carga viscosa y la FBC. Se graficó la FBC promedio una vez alcanzado el nuevo valor basal de FBC posdextrano. 


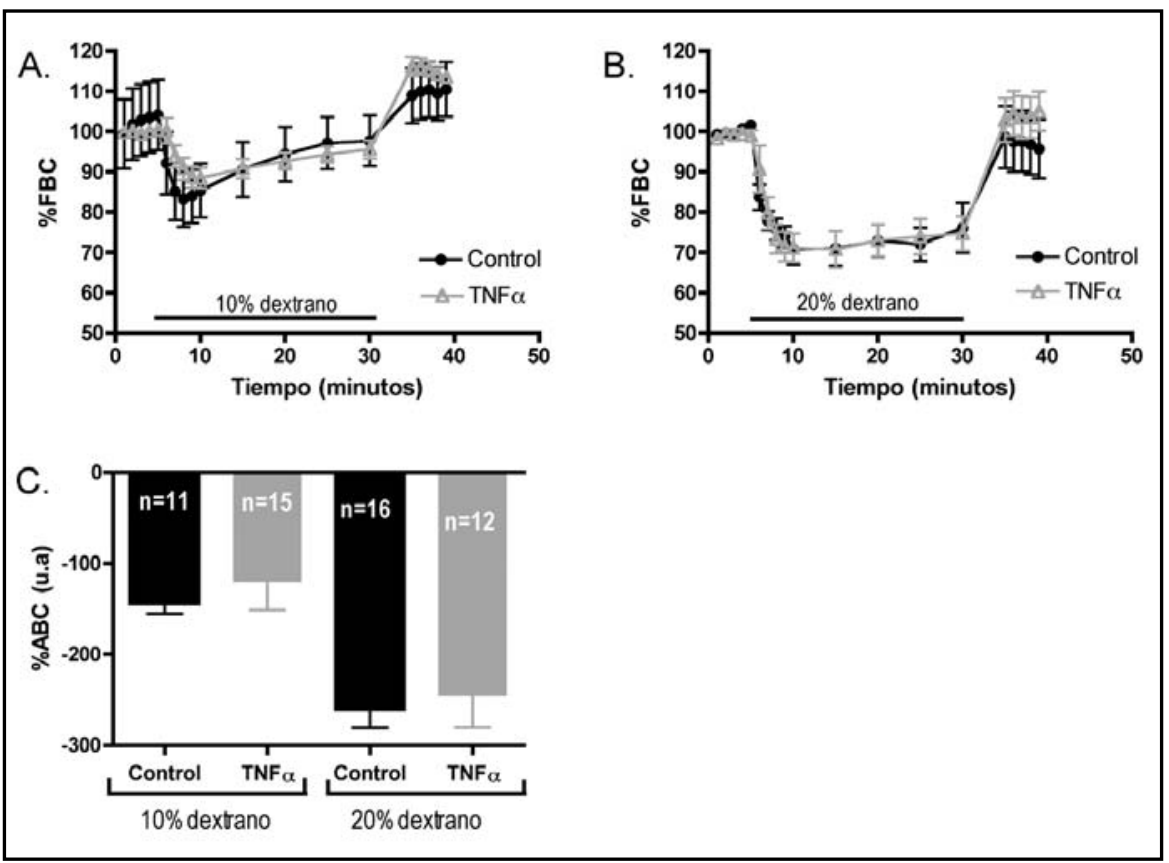

Figura 3. El tratamiento con TNF $\alpha$ por 24 horas no modifica la respuesta de FBC a la carga viscosa.

Curso temporal de los cambios en la FBC luego de 24 horas de tratamiento con TNF $\alpha$ (10 ng/ml) o solución control (HBSS, 1cP) expuestos a: A) dextrano 10\% (14,4cP) o B) dextrano 20\% (73cP). C) Área bajo la curva de la relación entre FBC (Figuras $A$ y $B$ ) y el tiempo en respuesta a dextrano $10 \%$ y $20 \%$. Cada barra representa el promedio $\pm \mathrm{EE}$.

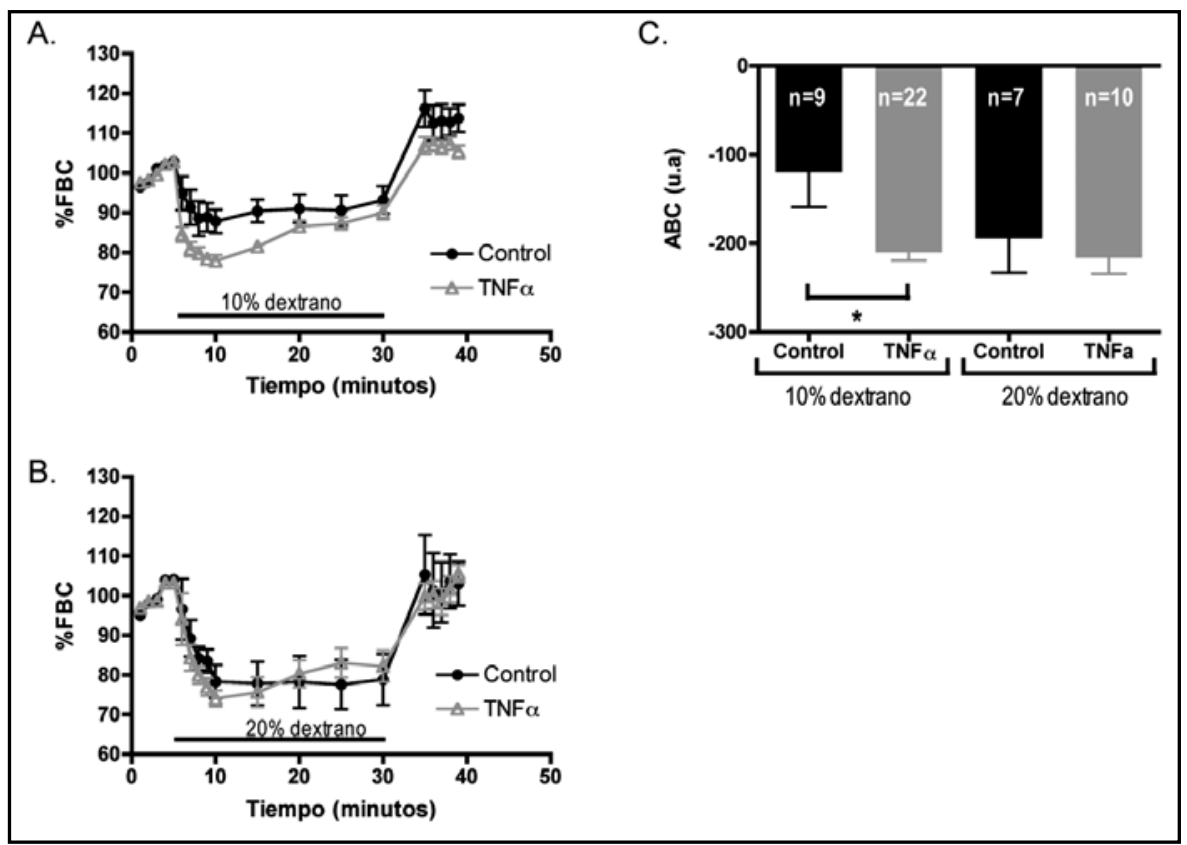

Figura 4. El tratamiento con TNF $\alpha$ por 48 horas disminuye la FBC en respuesta a baja viscosidad.

Curso temporal de los cambios en la FBC luego de 48 horas de tratamiento con TNF $\alpha(10 \mathrm{ng} / \mathrm{ml})$ o solución control (HBSS, $1 \mathrm{cP})$ expuestos a: A) dextrano 10\% $(14,4 \mathrm{cP})$ o B) dextrano $20 \%(73 \mathrm{cP})$. C) Área bajo la curva de la relación entre FBC (Figuras $A$ y $B$ ) y el tiempo en respuesta a dextrano $10 \%$ y $20 \%$. Cada barra representa el promedio \pm EE La disminución de la FBC y del área bajo la curva es significativamente mayor en las células tratadas con TNF $\alpha$ y expuestas a dextrano 10\%. ${ }^{*} p<0,05$. 
en cultivos tratados. Sin embargo el área bajo la curva fue significativamente mayor en los cultivos tratados con TNF $\alpha$ (Figura 5b).

Para evaluar el origen del $\left[\mathrm{Ca}^{2+}\right]_{\text {ic }}$ involucrado en esta respuesta se agregó el bloqueador de la bomba de calcio del retículo endoplásmico tapsigargina $(2,5 \mu \mathrm{M})$ a cultivos tratados y controles previo a la exposición a dextrano $10 \%$. Se observó una caída máxima en la FBC de $33,43 \% \pm 7,6$ en los cultivos tratados con TNF $\alpha$ (48 horas) y tapsigargina, con un área bajo la curva significativamente menor que los cultivos tratados solamente con TNF $\alpha$ (Figura 6a). Los cultivos controles tratados con tapsigargina presentaron una disminución máxima en la FBC de $14,75 \% \pm 2,19$. No se observó diferencia significati- va en el área bajo la curva en comparación con los controles sin bloqueo (Figura 6b).

\section{DISCUSIÓN}

Nuestros resultados sugieren que el mecanismo de autorregulación de las células ciliadas frente a la carga viscosa descrito en otros sistemas, se encuentra presente también en las células ciliadas de vía aérea superior. Este mecanismo permite al epitelio adaptar su FBC a diferentes viscosidades, evitando el colapso del transporte mucociliar. Observamos que cultivos de células ciliadas tratados con una citoquina proinflamatoria (TNF $\alpha)$ por 48 horas, en condiciones de baja viscosidad (dextrano
A.

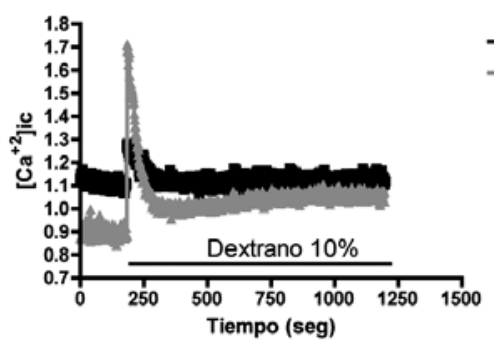

B.

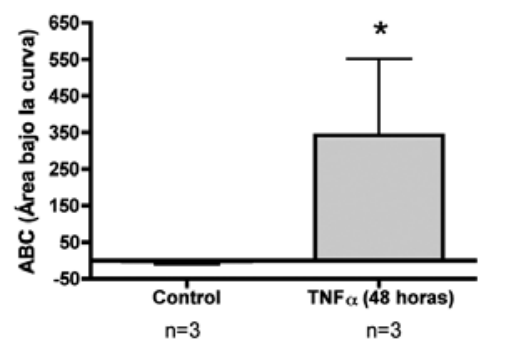

Figura 5. El tratamiento por 48 horas con TNF $\alpha$ incrementa los niveles de calico intracelular en respuesta al estímulo con dextrano $10 \%$.

A) Curso temporal de la concentración de Ca+2 intracelular expresado como intensidad de fluorescencia en función del tiempo. B) Área bajo la curva de la relación entre la concentración de $\mathrm{Ca}^{+2}$ y el tiempo (Figura A). ${ }^{\star} p<0,05$.

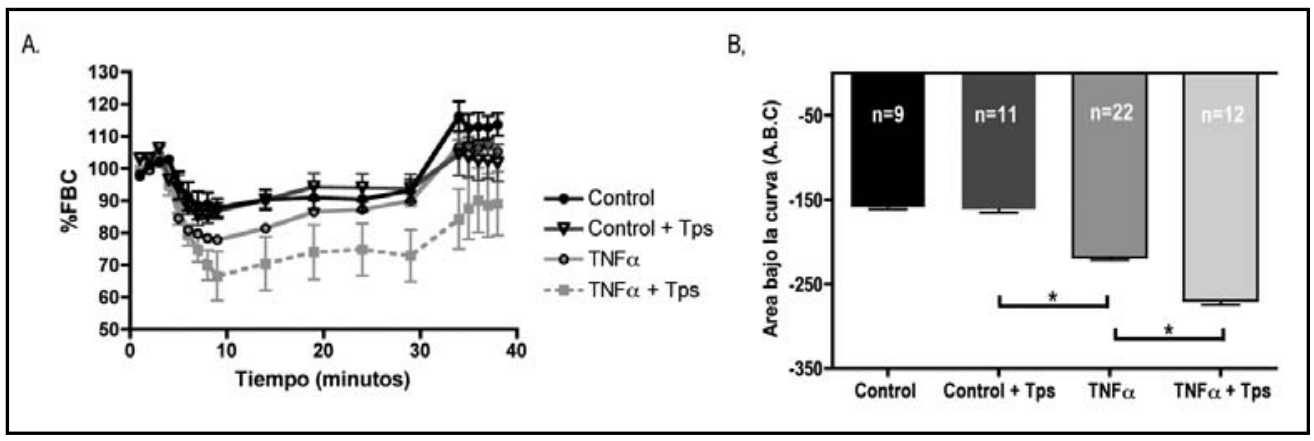

Figura 6. El calcio intracelular está involucrado en la respuesta de FBC a baja viscosidad luego del tratamiento por 48 horas con TNF $\alpha$. A) Curso temporal de los cambios en la FBC en cultivos tratados por 48 horas con TNF $\alpha$ ( $10 \mathrm{ng} / \mathrm{ml}$ ) o solución control (HBSS) expuestos a dextrano $10 \%$ en presencia 0 ausencia de tapsigargina $2,5 \mu \mathrm{M}$ (bloqueador de la bomba $\mathrm{Ca}^{+2} \mathrm{ATPase}$ reticular). B) Área bajo la curva de la relación entre FBC y tiempo (Figura A). Cada barra representa el promedio \pm EE para los diferentes tratamientos. *Diferencia estadísticamente significativa $(p<0,05)$. Observe que luego de la exposición a dextrano 10\%, la FBC de los cultivos controles no es afectada por el tratamiento con tapsigargina, mientras que los cultivos tratados con TNF $\alpha$ (48 horas) mostraron una mayor disminución en la FBC luego del tratamiento con tapsigargina. 
$10 \%$ ) reducen significativamente la FBC. Estos cambios se correlacionan con alteraciones en la respuesta del $\left[\mathrm{Ca}^{2+}\right]_{\mathrm{ic}}$.

El aumento de la viscosidad del medio externo provoca una disminución en la FBC de los cilios que transportan moco en diversos sistemas ${ }^{6,18}$. Nuestros resultados son similares a lo descrito en el epitelio de tráquea de conej $0^{5}$ y en epitelio de oviducto ${ }^{5,6}$. En condiciones de baja viscosidad (dextrano 10\%) se produce una disminución de la FBC importante, mientras que intervalos mayores de viscosidad (dextrano 20\% y $25 \%$ ) la FBC disminuye levemente o permanece relativamente constante. Andrade y col, demostraron que los cambios en la viscosidad del medio externo se asocian a cambios en $\left[\mathrm{Ca}^{2+}\right]_{\mathrm{ic}}$, sugiriendo que el calcio participa como señal que acopla las variaciones en la viscosidad del medio externo con las modificaciones en la $\mathrm{FBC}^{6}$. Además demostró que las modificaciones de la FBC a baja viscosidad son dependientes de los depósitos de $\left[\mathrm{Ca}^{2+}\right]_{\mathrm{ic}}$, mientras que a alta viscosidad se gatilla el mecanismo de regulación dependiente del calcio extracelular.

El factor de necrosis tumoral alfa (TNF $\alpha$ ) es una citoquina proinflamatoria producida por varios tipos celulares incluyendo células epiteliales y macrófagos. Se han encontrado niveles elevados de expresión de genes de TNF $\alpha$ en pacientes con sinusitis crónica hiperplástica comparado con controles $^{19}$ y niveles elevados de esta citoquina en mucosa nasal de pacientes con sinusitis crónica comparado con controles ${ }^{20}$. Por otra parte la persistencia de niveles elevados de mARN de TNF $\alpha$, además de otras citoquinas (IL-1beta, IL-6, IL-8, MCP-1) se asoció a persistencia de alteraciones en la tomografía de cavidades paranasales y persistencia de alteraciones endoscópicas en pacientes con sinusitis crónica ${ }^{10}$. Estos hallazgos sugieren un rol importante de esta citoquina en la patogenia de esta enfermedad.

Existen dos subtipos predominantes de receptores para TNF $\alpha$, p55TNF o TNFR1 y p75TNF 0 TNFR2. La gran mayoría de las respuestas conocidas de TNF $\alpha$ ocurren por activación de TNFR1 ${ }^{21}$. Nuestros cultivos mostraron inmunorreactividad positiva en el citoplasma de las células ciliadas para TNFR1 en condiciones basales, no así para TNFR2, posiblemente porque este último receptor se expresa en menor cantidad.
En enfermedades crónicas de vía aérea superior e inferior como el caso de la rinosinusitis crónica se ha observado un aumento en la viscosidad de las secreciones mucosas con un promedio de 16 poise ${ }^{22}$. Sin embargo no existen estudios in vitro que evalúen el efecto de la suma de niveles elevados de una citoquina proinflamatoria y aumento de la carga viscosa sobre la FBC de las células del epitelio respiratorio.

Nuestros resultados muestran que la FBC presenta una caída significativamente mayor en respuesta a baja viscosidad cuando las células han sido expuestas por 48 horas a TNF $\alpha$. No se observó alteración en la respuesta de FBC a viscosidades más altas ni con menos tiempo de exposición a esta citoquina. Esto se asoció a $\left[\mathrm{Ca}^{2+}\right]_{i \mathrm{c}}$ significativamente más altos en los cultivos pretratados con TNF $\alpha$. Estos efectos son similares a lo descrito en epitelio respiratorio humano en fibrosis quística, en que se observó que agentes proinflamatorios aumentan el tamaño de los depósitos intracelulares y la respuesta de calcio ${ }^{23,24}$. Considerando que la respuesta de la FBC a baja viscosidad depende de los depósitos de $\left[\mathrm{Ca}^{2+}\right]_{\mathrm{ic}}$, planteamos como hipótesis que el tratamiento con TNF $\alpha$ modifica la homeostasis del calcio celular proveniente de los depósitos intracelulares.

En vía aérea inferior diferentes autores han demostrado el rol de TNF $\alpha$ en modular la hiperreactividad de vía aérea alterando la homeostasis del calcio en las células del músculo liso, en que el pretratamiento con TNF $\alpha$ facilita la movilización de $\left[\mathrm{Ca}^{2+}\right]_{\mathrm{ic}}$ inducida por broncoconstrictores, efecto que probablemente involucra la expresión de proteínas ${ }^{25}$. En células de músculo liso traqueal se demostró que TNF $\alpha$ mediante la activación del R1TNF $\alpha$ estimula la expresión de ICAM 1 por una vía de señalización sensible a tapsigargina ${ }^{26}$ (depleta los depósitos de $\left[\mathrm{Ca}^{2+}\right]_{\mathrm{ic}}$ ). Estos hallazgos indican que la expresión de genes mediada por TNF $\alpha$ en células musculares lisas es íntimamente dependiente de los depósitos de $\left[\mathrm{Ca}^{2+}\right]_{\text {ic }}$ que pueden ser depletados por tapsigargina. En nuestro estudio al depletar los depósitos de calcio intracelular, las células pretratadas con TNF $\alpha$ presentaron una caída en la FBC en respuesta al incremento en la viscosidad del medio con dextrano $10 \%$, significativamente mayor que las células no tratadas con tapsigargina y 
significativamente mayor que las células controles (sin tratamiento con TNF $\alpha$ ). En las células pretratadas 48 horas con TNF $\alpha$ la respuesta de la $\mathrm{FBC}$ y los mayores niveles de $\left[\mathrm{Ca}^{2+}\right]_{\text {ic }}$ observados en respuesta a baja viscosidad, sugieren que TNF $\alpha$ parece modificar el mecanismo de respuesta a la viscosidad del medio externo haciendo a las células dependientes de los depósitos de calcio intracelular. Estos resultados apoyan la idea que epitelios expuestos a citoquinas presentan una hiperreactividad en el retículo endoplásmico liso ${ }^{24}$.

En condición control 0 en ausencia de citoquinas proinflamatorias, es posible que en el epitelio respiratorio humano la exposición a baja viscosidad (dextrano 10\%) no dependa del $\left[\mathrm{Ca}^{2+}\right]_{\text {ic }}$ de la misma manera que lo descrito para células del oviducto. Es probable que el mecanismo de control de FBC frente a variaciones de viscosidad obedezca a un mecanismo mixto, dependiente de depósitos de $\left[\mathrm{Ca}^{2+}\right]_{\text {ic }}$ y de influjo de calcio extracelular. Al observar el gráfico de FBS versus viscosidad (Figura 2B), la viscosidad asociada a dextrano 10\% se ubica en el punto de inflexión de la curva, entre caída significativa de FBC y la activación de un mecanismo de autorregulación observado para viscosidades mayores.

En conclusión nuestros resultados demuestran que los cultivos de células ciliadas respiratorias humanas expresan los receptores de TNF $\alpha \mathrm{R} 1$ y R2. Además la exposición a TNF $\alpha$ por 48 horas afecta la respuesta de la FBC frente a cambios en la viscosidad, probablemente alterando la homeostasis del calcio intracelular por un mecanismo dependiente de depósitos intracelulares sensibles a tapsigargina. Es necesario realizar estudios adicionales para establecer el efecto que procesos inflamatorios crónicos pueden tener sobre los mecanismos de control del transporte mucociliar, a nivel de las vías de señalización molecular y sus implicancias clínicas.

Fuente de apoyo económico:

1. Fondos Investigación Sociedad Chilena de Otorrinolaringología, Medicina y Cirugía de Cabeza y Cuello.

2. Fondecyt 1080679.

\section{BIBLIOGRAFÍA}

1. VAN DER BAAN B. Ciliary function. Acta Otorhinolaryngol Belg 2000; 54(3): 293-8.

2. Seybold ZV, Mariassy at, Stroh D, Kim CS, Gazeroglu $H$ and Wanner A. Mucociliary interaction in vitro: effects of physiological and inflammatory stimuli. J Appl Physiol 1990; 68(4): 1421-6.

3. Salathe M. Regulation of mammalian ciliary beating. Annu Rev Physiol 2007; 69: 401-22.

4. CoLE P. The damaging role of bacteria in chronic lung infection. J Antimicrob Chemother 1997; 40 Suppl A: 5-10.

5. Johnson NT, Villalon M, Royce fH, Hard R and VeRDUGo P. Autoregulation of beat frequency in respiratory ciliated cells. Demonstration by viscous loading. Am Rev Respir Dis 1991; 144(5): 1091-4.

6. Andrade YN, Fernandes J, Vázquez E, et al. TRPV4 channel is involved in the coupling of fluid viscosity changes to epithelial ciliary activity. $J$ Cell Biol 2005; 168(6): 869-74.

7. Bachert C, van Kempen MJ, Hopken K, Holtappels G and Wagenmann M. Elevated levels of myeloperoxidase, pro-inflammatory cytokines and chemokines in naturally acquired upper respiratory tract infections. Eur Arch Otorhinolaryngol 2001; 258(8): 406-12.

8. Bensch GW, Nelson HS and Borish LC. Evaluation of cytokines in nasal secretions after nasal antigen challenge: lack of influence of antihistamines. Ann Allergy Asthma Immunol 2002; 88(5): 457-62.

9. Repka-Ramírez S, Naranch K, Park YJ, Clauw D AND BARANIUK JN. Cytokines in nasal lavage fluids from acute sinusitis, allergic rhinitis, and chronic fatigue syndrome subjects. Allergy Asthma Proc 2002; 23(3): 185-90.

10. Kuehnemund M, Ismail C, Brieger J, Schaefer D AND Mann WJ. Untreated chronic rhinosinusitis: a comparison of symptoms and mediator profiles. Laryngoscope 2004; 114(3): 561-5.

11. Chen JH, Takeno S, Osada R, Ueda T and Yauin K. Modulation of ciliary activity by tumor necrosis factor-alpha in cultured sinus epithelial cells. Possible roles of nitric oxide. Hiroshima $\mathrm{J} M e d$ Sci 2000; 49(1): 49-55. 
12. Rhee CS, Hong SK, Min YG, et al. Effects of IL-1 beta, TNF-alpha, and TGF-beta on ciliary beat frequency of human nasal ciliated epithelial cells in vitro. Am J Rhinol 1999; 13(1): 27-30.

13. Verdugo P, Rumery Re and Tam PY. Hormonal control of oviductal ciliary activity: effect of prostaglandins. Fertil Steril 1980; 33(2): 193-6.

14. González C, Sánchez T, Fonseca X and Villalón M. Cultivo primario de células ciliadas de adenoides humanos: Un modelo experimental para evaluar la actividad ciliar in vitro. Rev Otorrinolaringol Cir Cabeza Cuello 2007; 67(2): 99-107.

15. Abdo M, Hisheh S, Arfuso $F$ and Dharmarajan $A$. The expression of tumor necrosis factor-alpha, its receptors and steroidogenic acute regulatory protein during corpus luteum regression. Reprod Biol Endocrinol 2008; 6: 50.

16. Villalon M and Verdugo P. Control of ciliary movement in mammalian oviductal ciliated cells. Arch Biol Med Exp (Santiago) 1992; 24: 344-50.

17. Barrera NP, Morales B and Villalon M. Plasma and intracellular membrane inositol 1,4,5trisphosphate receptors mediate the $\mathrm{Ca}\left({ }^{2+}\right)$ increase associated with the ATP-induced increase in ciliary beat frequency. Am J Physiol Cell Physiol 2004; 287(4): C1114-24.

18. Gheber L, Korngreen A and Priel Z. Effect of viscosity on metachrony in mucus propelling cilia. Cell Motil Cytoskeleton 1998; 39(1): 9-20.

19. Anand VK, Kacker A, Orjuela AF, Huang C, Manarey $C$ and Xiang J. Inflammatory pathway gene expression in chronic rhinosinusitis. $A m \mathrm{~J}$ Rhinol 2006; 20(4): 471-6.

20. Lennard CM, Mann EA, Sun LL, Chang AS and Bolger WE. Interleukin-1 beta, interleukin-5, interleukin-6, interleukin-8, and tumor necrosis factor-alpha in chronic sinusitis: response to systemic corticosteroids. Am J Rhinol 2000; 14(6): 367-73.

21. Wiegmann K, Schutze S, Kampen E, Himmler A, Machleidt T and Kronke M. Human 55-kDa receptor for tumor necrosis factor coupled to signal transduction cascades. J Biol Chem 1992; 267(25): 17997-8001.

22. Majima Y, Harada T, Shimizu T, et al. Effect of biochemical components on rheologic properties of nasal mucus in chronic sinusitis. Am J Respir Crit Care Med 1999; 160(2): 421-6.

23. Ribeiro CM, Paradiso AM, Carew MA, Shears SB AND Boucher RC. Cystic fibrosis airway epithelial $\mathrm{Ca}^{2+}$ i signaling: the mechanism for the larger agonist-mediated $\mathrm{Ca}^{2+} \mathrm{i}$ signals in human cystic fibrosis airway epithelia. J Biol Chem 2005; 280(11): 10202-9.

24. RibeiRo CM. The role of intracellular calcium signals in inflammatory responses of polarised cystic fibrosis human airway epithelia. Drugs RD 2006; 7(1): 17-31.

25. Amrani Y, Krymskaya V, Maki C and Panettieri RA, JR. Mechanisms underlying TNF-alpha effects on agonist-mediated calcium homeostasis in human airway smooth muscle cells. Am J Physiol 1997; 273(5 Pt 1): L1020-8.

26. Amrani Y, Lazaar AL, Hoffman R, Amin K, Ousmer $S$ and Panettierl RA, JR. Activation of p55 tumor necrosis factor-alpha receptor-1 coupled to tumor necrosis factor receptorassociated factor 2 stimulates intercellular adhesion molecule-1 expression by modulating a thapsigargin-sensitive pathway in human tracheal smooth muscle cells. Mol Pharmacol 2000; 58(1): 237-45.

Dirección: Dra. Claudia González G.

Marcoleta $3502^{\circ}$ piso, Santiago, Chile E mail: cgonzaga@puc.cl 